\title{
DESENVOLVIMENTO E VALIDAÇÃO DE MÉTODO PARA DETERMINAÇÃO DE BISFENOL A E ETINILESTRADIOL EM AGUAPÉ E MINI-PAPIRO PROVENIENTES DE WETLANDS CONSTRUÍDAS
}

\author{
Julyenne M. Camposa,*, (i), Denis M. Roston ${ }^{\mathrm{a}}$ e Sonia C. N. Queiroz ${ }^{\mathrm{b}, \#}$ \\ ${ }^{a}$ Faculdade de Engenharia Agrícola, Universidade Estadual de Campinas, 13083-875 Campinas - SP, Brasil

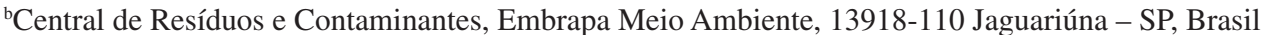

Recebido em 20/02/2019; aceito em 09/05/2019; publicado na web em 30/08/2019

\begin{abstract}
DEVELOPMENT AND VALIDATION OF METHOD FOR DETERMINATION OF BISPHENOL A AND ETHINYLESTRADIOL IN WATER HYACINTH AND DWARF PAPYRUS FROM CONSTRUCTED WETLANDS. Bisphenol A and ethinylestradiol, both considered as endocrine disruptors, have been found in surface water and effluents from municipal wastewater supply and can cause adverse health effects on wildlife species and in human beings at extremely low level. In order to remove these compounds from wastewater, constructed wetlands has been used since it proved to be more efficient, and has less cost than conventional treatments. One of the mechanisms of removal of endocrine disruptors by constructed wetlands is the uptake by macrophytes, so it is necessary to analyze these compounds in the plants of the system. Thus, the objective of this study was to develop and validate two methods by high performance liquid chromatography with fluorescence detector capable of determining these compounds in dwarf papyrus and water hyacinth. The evaluated parameters in the validation were: linearity $\left(\mathrm{R}^{2} \geq 0.99\right)$, limit of detection $\left(\mathrm{LOD}=0.045-0.15 \mu \mathrm{g} \mathrm{g}^{-1}\right)$, limit of quantification ( $\mathrm{LOQ}=0.15-1.50 \mu \mathrm{g} \mathrm{\textrm {g } ^ { - 1 }}$ ), accuracy (recoveries from 83.5 to $119.4 \%$ ) and precision (intra-day $\mathrm{CV} \leq 6.2 \%$ and inter-day $\mathrm{CV} \leq 5.8 \%$ ). Samples from a constructed wetland were analyzed and the developed methods showed be suitable for intended purpose.
\end{abstract}

Keywords: Cyperus isocladus; Eichhornia crassipes; wastewater; liquid chromatography; endocrine disruptors.

\section{INTRODUÇÃO}

Interferentes endócrinos (IE) compreendem um amplo espectro de substâncias naturais e sintéticas, ${ }^{1}$ e são definidos pela Agência de Proteção Ambiental Americana (USEPA) como "um agente exógeno que interfere na síntese, secreção, transporte, ligação, ação ou eliminação dos hormônios naturais no corpo que são responsáveis pela manutenção da homeostase, reprodução, desenvolvimento e/ou comportamento". ${ }^{2}$ Não existe uma classificação padronizada mundialmente que define quais substâncias são ou não interferentes endócrinos. Os produtos farmacêuticos e de higiene pessoal pertencem a um grupo de IEs já bem conhecido e definido, mas existem outros compostos químicos que podem interferir no funcionamento endócrino de seres humanos e animais que ainda não foram testados, ou as informações disponíveis sobre os efeitos adversos estão incompletas ou são controversas. ${ }^{3}$

O bisfenol A (BPA) é introduzido no meio aquático por meio de efluentes municipais e industriais continuamente. ${ }^{4}$ Devido à migração do BPA do plástico de mamadeiras e embalagens de alimentos, alguns países regulamentaram o uso de BPA nesses tipos de embalagens, especialmente em mamadeiras, ${ }^{1}$ sendo o Canadá o primeiro país a considerar o BPA como substância tóxica e estabelecer legislações para este composto. ${ }^{4}$ No Brasil a Agência Nacional de Vigilância Sanitária proibiu o uso de BPA na fabricação de mamadeiras em 2012. ${ }^{5}$

Foram encontradas concentrações de BPA em águas residuárias e estações de tratamento de esgoto variando de 100 a $6.900 \mathrm{ng} \mathrm{L}^{-1}$ na Ásia, ${ }^{6}$ concentrações de até $84.110 \mathrm{ng} \mathrm{L}^{-1}$ no Brasil, ${ }^{7}$ e de até $1.550 \mathrm{ng} \mathrm{L}^{-1} \mathrm{em}$ Portugal. ${ }^{8}$ Em águas superficiais no Rio Atibaia (no estado de São Paulo, Brasil), concentrações de 520 a $6.375 \mathrm{ng} \mathrm{L}^{-1}$ de BPA foram encontradas. ${ }^{9}$

\footnotetext{
*e-mail: julyenne.mc@ gmail.com

"alternative e-mail: sonia.queiroz@embrapa.br
}

O etinilestradiol (EE2) é considerado um dos principais contaminantes das águas residuárias, porque foi encontrado em águas superficiais e em efluentes provenientes de águas de abastecimento municipais em concentrações na ordem de nanogramas, capazes de causar alterações no sistema reprodutor de peixes e no metabolismo de organismos aquáticos. ${ }^{10-12}$

No Brasil, EE2 foi encontrado em águas residuárias em concentrações de até $1260 \mathrm{ng} \mathrm{L}^{-1},{ }^{7}$ enquanto em países como Austrália, Reino Unido e Alemanha concentrações máximas de $2 \mathrm{ng} \mathrm{L}^{-1}$ foram relatadas. ${ }^{13-15}$

Em águas superficiais dos Estados Unidos foram encontradas concentrações variando de 3 a $273 \mathrm{ng} \mathrm{L}^{-1}$ de EE2,${ }^{16}$ enquanto no Brasil foram encontradas concentrações de 501 a $4.390 \mathrm{ng} \mathrm{L}^{-1} \cdot{ }^{9} \mathrm{Na}$ Itália foram encontradas concentrações de até $34 \mathrm{ng} \mathrm{L}^{-1},{ }^{17}$ e na Alemanha de no máximo $1 \mathrm{ng} \mathrm{L^{-1 }} .^{15}$

A remoção total destes compostos em estações convencionais de tratamento de esgoto não ocorre, uma vez que estas substâncias foram detectadas em águas superficiais e residuárias, conforme citado anteriormente. ${ }^{6-9,13-15}$ Outra fonte de contaminação ocorre nos locais onde o esgoto é jogado in natura nos corpos d'água. Alguns sistemas de tratamento tais como os que envolvem processos oxidativos avançados (POA) ${ }_{1}^{18}$ osmose reversa, ${ }^{3}$ nanofiltração, ${ }^{3}$ carvão ativado, ${ }^{3}$ biorreatores de membrana, ${ }^{3}$ e wetlands construídas, ${ }^{18}$ são mais eficientes na redução desses compostos do que os métodos tradicionais utilizados nas estações de tratamento de esgoto (como lodo ativado, por exemplo). ${ }^{18,3}$

Pesquisadores buscam diferentes tecnologias de remoção destes compostos pelo fato de algumas destas tecnologias avançadas (POA, osmose reversa, nanofiltração, biorreatores de membrana) disponíveis possuírem limitações técnicas relacionadas ao tipo de afluente, alto custo, ou não removerem os compostos por completo. ${ }^{19}$ No entanto, geralmente os métodos mais eficientes não são adequados para implementação no campo ou em comunidades rurais e isoladas. ${ }^{20}$ 
O tratamento realizado por wetlands construídas baseia-se nas interações que envolvem o solo, a vegetação e os micro-organismos presentes neste tipo de sistema, ${ }^{21}$ promovendo diversos processos físicos e biológicos como absorção pelas macrófitas, adsorção, precipitação, nitrificação, decomposição, e filtração física e biológica, que é realizada pelo biofilme bacteriano aderido ao meio suporte e raízes da macrófita. ${ }^{22}$ Devido a esses diferentes processos de tratamento realizados pelas wetlands construídas, a utilização deste tipo de sistema para remoção de interferentes endócrinos em águas residuárias têm se mostrado eficiente por diversos autores e são adequados para serem aplicados em pequenas propriedades. ${ }^{10,23-33}$

Tendo em vista que um dos mecanismos de remoção de interferentes endócrinos por wetlands construídas é a absorção destes pelas macrófitas, é de extrema importância analisar, além das concentrações destes na água residuária, também nas plantas, para que seja possível elucidar os mecanismos de remoção destes compostos nos diferentes compartimentos das wetlands construídas. Na Figura 1 estão apresentados os componentes que fazem parte de uma wetland construída de fluxo subsuperficial.

Pelo fato de não haver metodologias publicadas para identificação e quantificação de bisfenol A e etinilestradiol em macrófitas provenientes de wetlands construídas, o objetivo deste trabalho foi desenvolver e validar dois métodos por cromatografia líquida de alta eficiência (HPLC) com detector de fluorescência (FL) capazes de determinar estes compostos em macrófitas, provenientes de wetlands construídas.

\section{PARTE EXPERIMENTAL}

\section{Reagentes}

O hormônio etinilestradiol que foi adicionado ao esgoto sintético foi obtido por meio de pílulas anticoncepcionais maceradas (Ciclo $21^{\circledR}$ ) e o utilizado nos ensaios de validação possuía $\geq 99,7 \%$ de pureza adquirido da Sigma-Aldrich ${ }^{\circledR}$. O composto bisfenol A que foi utilizado tanto no esgoto sintético quanto nas curvas analíticas possuía $\geq 97 \%$ de pureza adquirido da Sigma-Aldrich ${ }^{\circledast}$.

Tanto o metanol $(\mathrm{MeOH})$ quanto a acetonitrila $(\mathrm{ACN})$ utilizados em todos os ensaios possuíam grau HPLC/UV e foram adquiridos da J. T. Baker ${ }^{\circledR}$. A água ultrapura foi obtida de um sistema Milli-Q ${ }^{\circledR}$ (modelo Advantage A10, Millipore, resistividade $=18,2 \Omega \mathrm{cm}$ ).

\section{Extração das amostras}

A análise de interferentes endócrinos no tecido vegetal foi realizada no Laboratório de Resíduos e Contaminantes (LRC) da Embrapa Meio Ambiente, localizado em Jaguariúna, SP.

As amostras foram oriundas de uma wetland construída em escala laboratorial que foi abastecida com esgoto sintético, simulando um esgoto municipal de carga média, com relação C:N:P de 200:50:8 durante dois anos. Após um ano e três meses do início do abastecimento foram adicionados a esse esgoto sintético o hormônio e o composto bisfenol A. Ao final do experimento as macrófitas foram coletadas das wetlands construídas.

As macrófitas livres de BPA e EE2 que foram utilizadas na etapa de validação foram retiradas de wetlands construídas sem injeção de interferentes endócrinos (controle). Para a realização deste ensaio as amostras foram previamente secas em estufa (marca FANEM, modelo 320 -SE) à $65^{\circ} \mathrm{C}$ até peso constante, de acordo com metodologia descrita na literatura, ${ }^{35} \mathrm{e}$ foram trituradas em moinho de facas (Marconi TE340). A seguir, foram pesados 2 gramas de amostra (1,5 gramas da parte aérea e 0,5 grama da raiz) em frasco erlenmeyer de vidro de $125 \mathrm{~mL}$ com tampa.

Foram adicionados à amostra $15 \mathrm{~mL}$ de metanol e $15 \mathrm{~mL}$ de acetonitrila, e em seguida os frascos colocados em agitador orbital, modelo Tecnal (TE-424), com temperatura controlada de $25^{\circ} \mathrm{C}$ com vidro de proteção ultravioleta. As amostras permaneceram sob agitação à 200 rpm por 2 horas. Após o período de agitação, as amostras foram filtradas em sistema à vácuo com membrana de fibra de vidro com diâmetro de poro de $1,2 \mu \mathrm{m}$.

As paredes do sistema de filtração e a macrófita sobre o filtro foram lavadas com $50 \mathrm{~mL}$ de acetonitrila, recolhendo-se esse solvente filtrado. Avolumaram-se os $50 \mathrm{~mL}$ de solvente para $500 \mathrm{~mL}$ com água, e a extração em fase sólida (EFS) ocorreu de acordo com o descrito na literatura, ${ }^{36}$ com algumas modificações, conforme a seguir.

$\mathrm{O}$ volume obtido de amostra $(500 \mathrm{~mL})$ foi percolado em um cartucho de EFS Hypersep ${ }^{\mathrm{TM}}$ Retain PEP da marca Thermo Scientific (6 mL e $250 \mathrm{mg}$ ). Porém, antes do uso, os cartuchos foram ativados com $5 \mathrm{~mL}$ de metanol e $7 \mathrm{~mL}$ de acetonitrila e condicionados com $5 \mathrm{~mL}$ de água ultrapura. A vazão da amostra no cartucho foi de 1 a $2 \mathrm{~mL} \mathrm{~min}^{-1}$.

O clean-up foi realizado com $15 \mathrm{~mL}$ de água ultrapura, e a eluição foi feita com 1,5 mL de metanol e 1,5 mL de acetonitrila em tubo concentrador, com posterior ajuste de volume para $3 \mathrm{~mL}$. As amostras foram filtradas e injetadas em triplicata no mesmo dia.

\section{Determinação cromatográfica}

As análises foram realizadas em um cromatógrafo líquido de alta eficiência (HPLC) Shimadzu Class VP constituído de sistema de injeção automática, bomba de alta pressão, forno para coluna e detector de fluorescência modelo RF-10AXL. As condições cromatográficas também foram adaptadas da literatura. ${ }^{36} \mathrm{~A}$ separação cromatográfica foi realizada utilizando a coluna tipo C18 da marca Phenomenex Gemini, $5 \mu \mathrm{m}, 110$ Å, 250 x 4,6 mm. A fase móvel foi acetonitrila e água ultrapura, a vazão foi de $0,8 \mathrm{~mL} \mathrm{~min}^{-1}$ e o volume de injeção de $50 \mu \mathrm{L}$ de amostra.

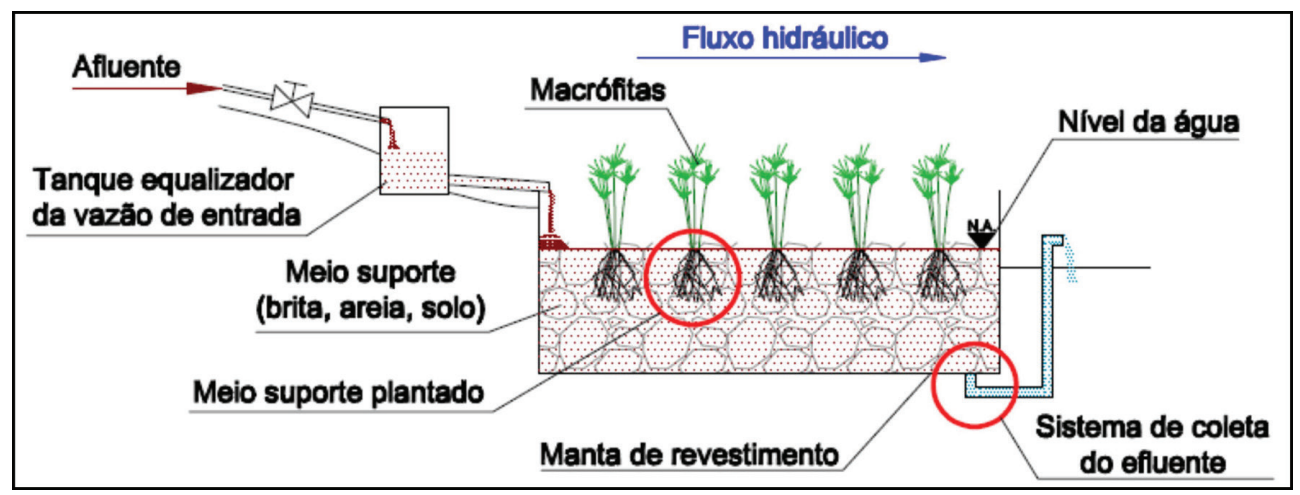

Figura 1. Representação esquemática dos componentes de uma wetland construída ${ }^{34}$ 


\section{Validação dos métodos cromatográficos}

Por ainda não existir uma metodologia oficial para análise de interferentes endócrinos em tecido vegetal por HPLC com detector de fluorescência, fez-se necessário o desenvolvimento e validação dos métodos analíticos, antes que fossem realizadas as análises das amostras.

As validações dos métodos cromatográficos foram realizadas separadamente para as duas plantas (aguapé e mini-papiro), utilizando-se plantas isentas dos IEs de interesse. Para isso, foi utilizado o DOQ-CGCRE-008 do Inmetro (2018), ${ }^{37}$ e também o SANTE/11813/2017 da Comissão Europeia (2018). ${ }^{38}$

A concentração de interferentes endócrinos em macrófitas provenientes de wetlands construídas são muito baixas, na faixa de $\mu \mathrm{g} \mathrm{g}^{-1} \mathrm{e}$ ng g ${ }^{-1},{ }^{39}$ consideradas como concentrações "traço", e os parâmetros de validação que devem ser avaliados para análise de traços são seletividade, linearidade e faixa linear de trabalho, limite de detecção e limite de quantificação, exatidão (recuperação), precisão (repetitividade e precisão intermediária). ${ }^{37}$

\section{Seletividade e efeito matriz}

A seletividade foi avaliada de maneira visual ao comparar três cromatogramas das matrizes (macrófita) sem os analitos (amostra branco), com três cromatogramas das matrizes com os analitos (amostra fortificada com $0,75 \mu \mathrm{g} \mathrm{g}^{-1}$ ), com o objetivo de verificar a presença de substâncias interferentes nos mesmos tempos de retenção dos analitos.

A avaliação da seletividade também foi realizada para os dois interferentes endócrinos, por meio de testes de efeito matriz. O efeito matriz (EM) foi calculado pela injeção de duas curvas analíticas com três diferentes concentrações dos compostos (baixa, média e alta), uma diluída na fase móvel e outra no extrato das plantas. As duas curvas foram comparadas, e então foram aplicados os testes $F$ de homogeneidade de variância com nível de confiança de $95 \%$ e 26 graus de liberdade (g. 1.), e então o teste $t$ de Student (duas amostras presumindo variâncias equivalentes), com nível de confiança de 95\% e 52 graus de liberdade ( 26 g. 1. da curva na fase móvel e 26 g. 1. da curva no extrato), utilizando o Microsoft Excel, de acordo com a literatura. ${ }^{37}$

\section{Limites de detecção e de quantificação}

Os limites de detecção (LDI) e de quantificação (LQI) instrumental (do HPLC) foram obtidos a partir da injeção de soluções de BPA e EE2 diluídas sucessivamente em fase móvel, observando qual a menor concentração dos analitos que seria identificada pelo equipamento, com precisão e exatidão aceitáveis. Foram consideradas aceitáveis as concentrações que corresponderam a um coeficiente de variação (CV) menor que $20 \%$ como LQI, e o menor ponto identificado com CV maior que $20 \%$ e com relação de área de sinal/ruído igual a três como LDI.

Foram considerados como limite de quantificação do método a menor concentração obtida nos ensaios de recuperação (com os extratos das plantas) que obteve precisão e exatidão aceitáveis; como limite de detecção do método a concentração que teve o pico identificado no cromatograma, porém a precisão e exatidão não obtiveram coeficientes de variação confiáveis.

\section{Linearidade, faixa linear de trabalho e sensibilidade}

A linearidade e a faixa linear de trabalho foram avaliadas de acordo com a literatura: ${ }^{37}$ verificando-se a presença de valores outliers pelo teste de Grubbs com auxílio do software Minitab $18^{\circledR}$, pela equação da regressão linear dos compostos, observando-se também a distribuição dos resíduos (calculados e representados graficamente com auxílio do Microsoft Excel $^{\circledR}$ ) e realizando-se a análise de variância (ANOVA) da regressão.

As curvas foram compostas de 7 concentrações, no aguapé de 0,038 a $3,75 \mu \mathrm{g} \mathrm{g}^{-1}$ para ambos compostos e no mini-papiro de 0,038 a 7,50 $\mu \mathrm{g} \mathrm{g} \mathrm{g}^{-1}$ para BPA. Apenas para o composto EE2 no mini-papiro a curva foi composta por 6 pontos, de 0,038 a 3,75 $\mu \mathrm{g} \mathrm{g}^{-1}$. Cada ponto das curvas analíticas foi injetado três vezes. A quantificação foi feita por padronização externa e a curva feita na fase móvel, tendo em vista que também foram realizados testes de efeito matriz. A sensibilidade foi calculada por meio da inclinação da curva analítica.

\section{Ensaios de recuperação}

A medida da exatidão do método foi avaliada por meio de ensaios de recuperação. A fortificação foi realizada em amostras de macrófitas isentas dos compostos (já previamente secas e moídas) com soluções de BPA e EE2 em acetonitrila. Após a adição dos analitos, as amostras foram processadas de acordo com a metodologia de análise e injetadas no cromatógrafo no mesmo dia.

No aguapé foram adicionadas as concentrações de $0,15 \mu \mathrm{g} \mathrm{g}^{-1}$, $0,75 \mu \mathrm{g} \mathrm{g}^{-1}$ e $1,5 \mu \mathrm{g} \mathrm{g}^{-1}$ para BPA, e $0,75 \mu \mathrm{g} \mathrm{g}^{-1}$ e $1,5 \mu \mathrm{g} \mathrm{g}^{-1}$ para EE2 (ambos com 3 ensaios cada nível, injetados em triplicata); no minipapiro os níveis utilizados com o composto BPA foram 1,5 e 3,0 $\mu \mathrm{g} \mathrm{g}^{-1}$ (3 ensaios para 1,5 $\mu \mathrm{g} \mathrm{g}^{-1}$ e 6 ensaios para 3,0 $\mu \mathrm{g} \mathrm{g}^{-1}$, ambos injetados em triplicata) e para EE2 foram $0,15 \mu \mathrm{g} \mathrm{g}^{-1}, 0,75 \mu \mathrm{g} \mathrm{g}^{-1}, 1,5 \mu \mathrm{g} \mathrm{g}^{-1}$ e 3,0 $\mu \mathrm{g} \mathrm{g}^{-1}$ (todos níveis com 3 ensaios, exceto para 3,0 $\mu \mathrm{g} \mathrm{g}^{-1}$ que obteve 6 ensaios, e ambos foram injetados em triplicata).

\section{Repetitividade e precisão intermediária}

A precisão foi obtida por meio da determinação do coeficiente de variação das replicatas no mesmo dia (repetitividade) e em dias diferentes (precisão intermediária). Para aguapé foram realizados três ensaios injetados em triplicata com os níveis $0,15 \mu \mathrm{g} \mathrm{g}^{-1}, 0,75 \mu \mathrm{g} \mathrm{g}^{-1}$ e $1,5 \mu \mathrm{g} \mathrm{g}^{-1}$, tanto para BPA quanto para EE2, enquanto que para mini-papiro foram realizados quatro ensaios injetados em triplicata nas concentrações de $0,15 \mu \mathrm{g} \mathrm{g}^{-1}, 0,75 \mu \mathrm{g} \mathrm{g}^{-1}, 1,5 \mu \mathrm{g} \mathrm{g}^{-1}$ e $3,0 \mu \mathrm{g} \mathrm{g}^{-1}$ para os dois IEs estudados.

Pelo fato de as amostras do ensaio de recuperação terem sido processadas e injetadas no mesmo dia, esses valores puderam ser considerados como repetitividade. ${ }^{38} \mathrm{~A}$ precisão intermediária foi obtida em dias diferentes.

\section{RESULTADOS E DISCUSSÃO}

\section{Otimização das condições de separação}

Os solventes utilizados como fase móvel foram acetonitrila (grau HPLC) e água ultrapura. A corrida cromatográfica iniciou na proporção de 50:50, v/v, no modo isocrático, por 21 minutos, mas após a passagem de algumas amostras verificou-se que apesar do preparo e limpeza da amostra ela estava causando instabilidade na linha de base. Assim, foi necessário incluir uma etapa de limpeza da coluna após cada injeção de amostra. Para isso, após a realização da eluição da fase móvel por 20 minutos na proporção 50:50 (v/v), aumentou-se a acetonitrila para $95 \%$ em 2 minutos e em seguida, manteve-se esta proporção por mais 8 minutos, retornando para $50 \%$ em 3 minutos, totalizando 33 min de corrida Após a adição desta etapa de limpeza observou-se que a linha de base se estabilizou.

\section{Seletividade}

A seletividade foi avaliada por comparação de cromatogramas das matrizes sem os analitos (amostra branco), com o cromatograma das matrizes contendo analitos (amostra fortificada), para verificar 
visualmente se existia algum interferente na matriz que saísse no mesmo tempo de retenção dos analitos de interesse. Observa-se na Figura 2 (aguapé) e na Figura 3 (mini-papiro) que nenhum interferente foi observado no mesmo tempo de retenção dos analitos (BPA e EE2).

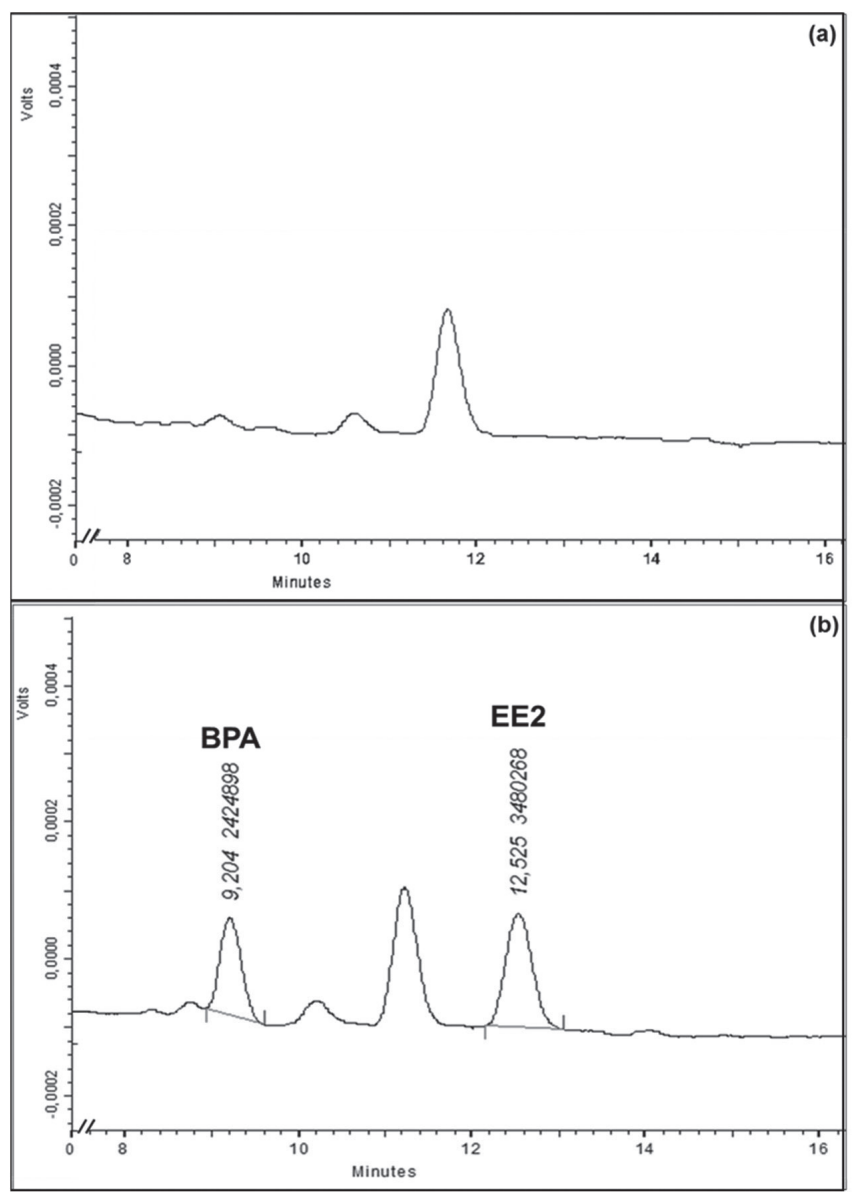

Figura 2. Cromatogramas para avaliação de seletividade do aguapé: (a) amostra branco e (b) amostra fortificada com BPA e EE2 na concentração de $0,75 \mu g g^{-1}$

De acordo com a Tabela 1 , todos os valores de $F$ calculados foram menores que $F$ tabelados, indicando assim que as variâncias puderam ser consideradas iguais e por isso os dados foram agrupados como variâncias equivalentes (26 g. 1. da fase móvel +26 g. 1. do extrato) e o teste $t$ de Student foi aplicado. Observa-se que todos os valores de $t$ calculado foram menores que de $t$ tabelado (Tabela 1) para 52 graus de liberdade, logo, foi possível afirmar que não houve efeito matriz para as amostras, conforme sugerido pelo documento do Inmetro. ${ }^{37}$ Desta maneira os métodos de análise de BPA e EE2 em amostras de mini-papiro e aguapé puderam ser considerados seletivos.

\section{Linearidade, faixa linear de trabalho e sensibilidade}

A linearidade e faixa linear de trabalho estão apresentadas na Figura 4 (mini-papiro) e na Figura 5 para o aguapé, onde é possível observar que houve linearidade para BPA e EE2, com coeficiente de determinação $\left(\mathrm{R}^{2}\right)$ maior que 0,99 para todos compostos e macrófitas, e com distribuição aleatória dos resíduos. A sensibilidade foi obtida através da inclinação da curva e observou-se que o método foi sensível, tendo em vista que um pequeno aumento na concentração do analito, ocasiona um aumento significativo na resposta do detector.

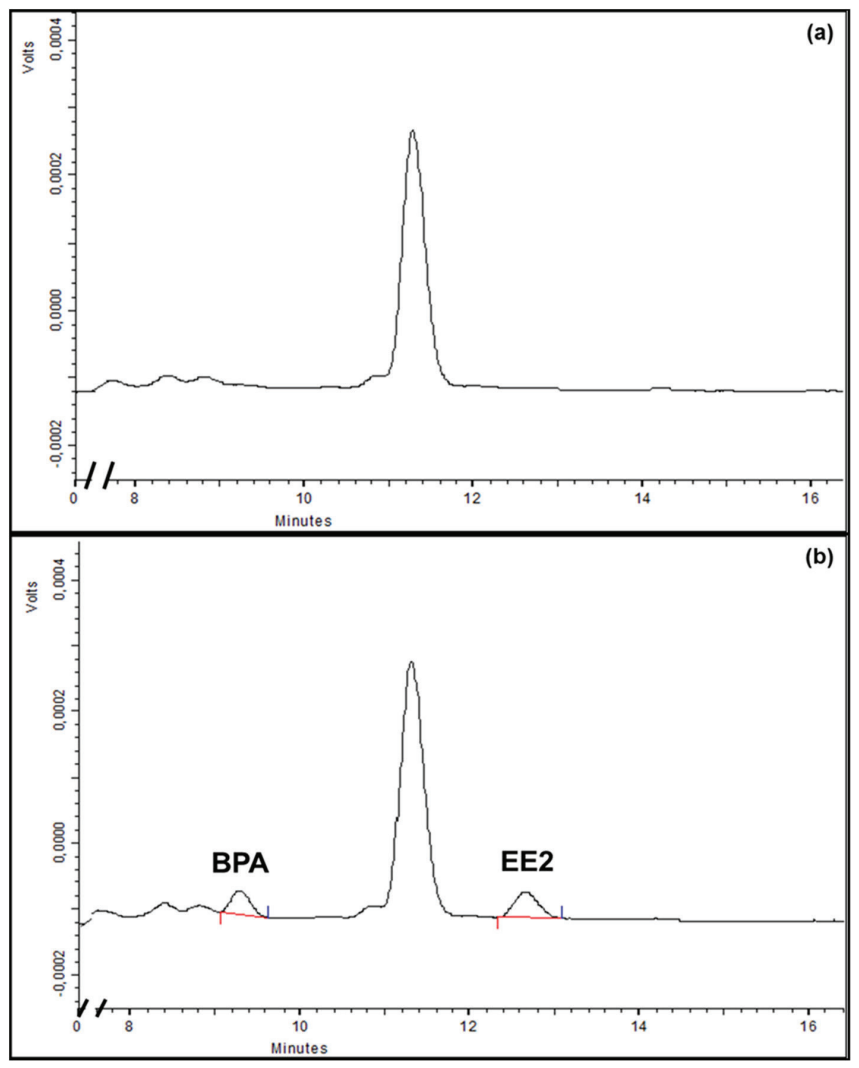

Figura 3. Cromatogramas de seletividade do mini-papiro: (a) amostra branco $e$ (b) amostra fortificada com BPA e EE2 na concentração de 0,75 $\mu \mathrm{g} \mathrm{g^{-1 }}$

Tabela 1. Valores do teste $F$ e do teste $t$ de Student para as amostras de aguapé e mini-papiro.

\begin{tabular}{lllll}
\hline & \multicolumn{2}{c}{ Aguapé } & \multicolumn{2}{c}{ Mini-papiro } \\
\cline { 2 - 5 } & BPA & EE2 & BPA & EE2 \\
\hline$F$ calculado & 1,37 & 1,01 & 1,51 & 1,29 \\
$F$ tabelado & 1,93 & 1,93 & 1,93 & 1,93 \\
$t$ calculado & 0,89 & 0,05 & 1,01 & 0,71 \\
$t$ tabelado & 1,67 & 1,67 & 1,67 & 1,67 \\
\hline
\end{tabular}

Não houve valores outliers de acordo com o teste de Grubbs. Na Tabela 2 pode-se observar a estatística da regressão, na Tabela 3 a ANOVA realizada para mini-papiro, e na Tabela 4 a ANOVA realizada para aguapé, para verificar a quantidade de resíduos do modelo, o ajuste da reta e a distribuição dos resíduos.

\section{Limites de detecção e de quantificação}

Os valores de LQI foram $0,038 \mu \mathrm{g} \mathrm{g}^{-1}$ para BPA e EE2, e os valores de LDI foram $0,023 \mu \mathrm{g} \mathrm{g}^{-1}$ para BPA e EE2. Os limites de detecção e de quantificação do método são apresentados na Tabela 5. Foram realizados três ensaios (réplicas) para cada limite (detecção ou quantificação) e injetados em triplicata.

Um método analítico para identificação e quantificação de 81 micropoluentes, dentre eles BPA e EE2, em $P$. australis proveniente de wetlands construídas utilizando extração prévia em micro-ondas, com posterior extração em fase sólida utilizando cartuchos Oasis HLB (similares ao Hypersep Retain PEP utilizados neste experimento), e leitura em UHPLC-MS/MS foi desenvolvido por Petrie et al. ${ }^{39}$ Foram obtidos LDI de $0,03 \mathrm{ng} \mathrm{mL}^{-1}$ para BPA e de $0,10 \mathrm{ng}$ $\mathrm{mL}^{-1}$ para EE2, e LQI de $0,10 \mathrm{ng} \mathrm{mL}^{-1}$ para BPA e $0,48 \mathrm{ng} \mathrm{mL}^{-1}$ 


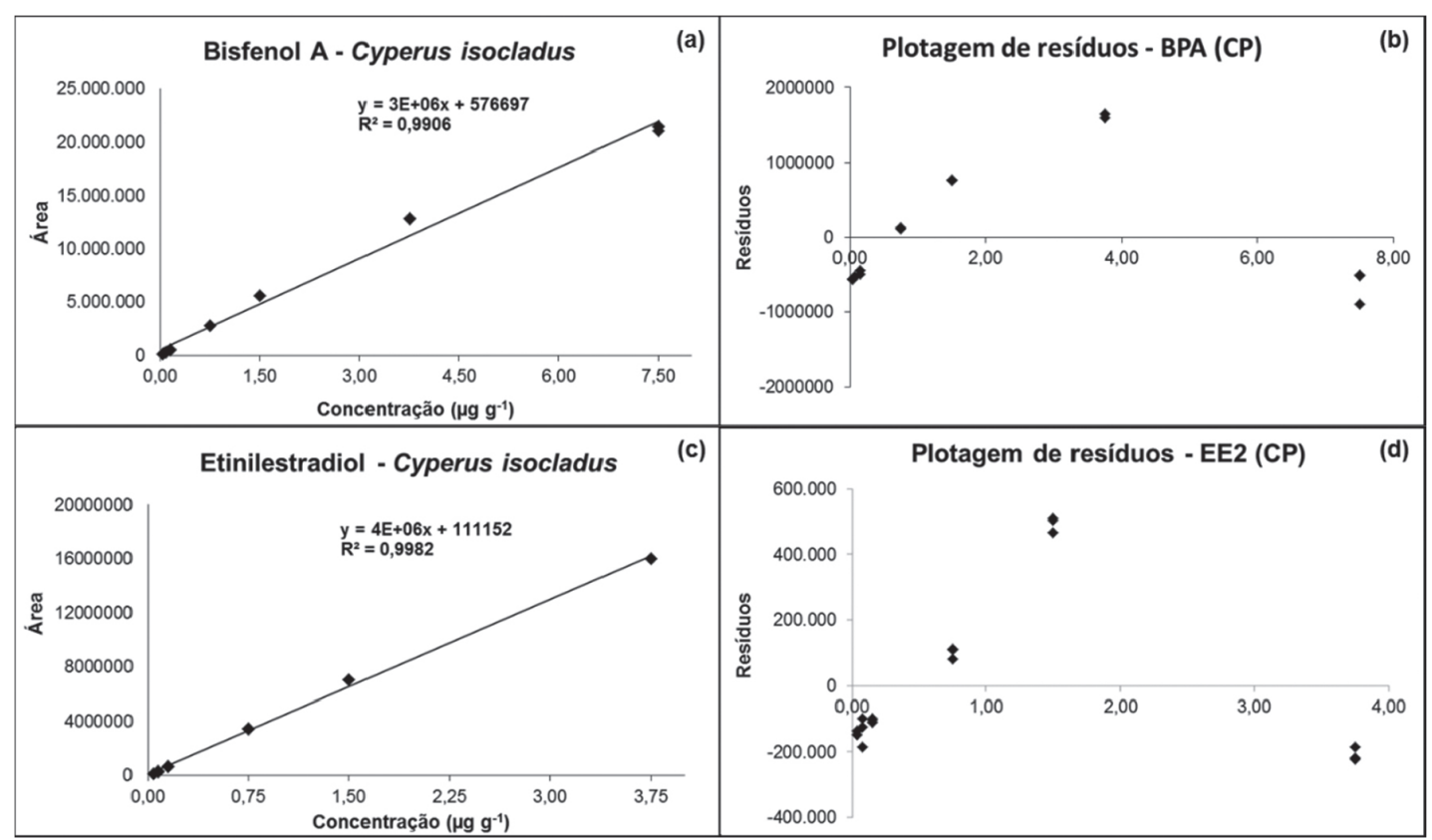

Figura 4. Curva analítica de BPA (a) e plotagem dos resíduos de BPA (b), e curva analítica de EE2 (c) e plotagem dos resíduos de EE2 (d) em mini-papiro

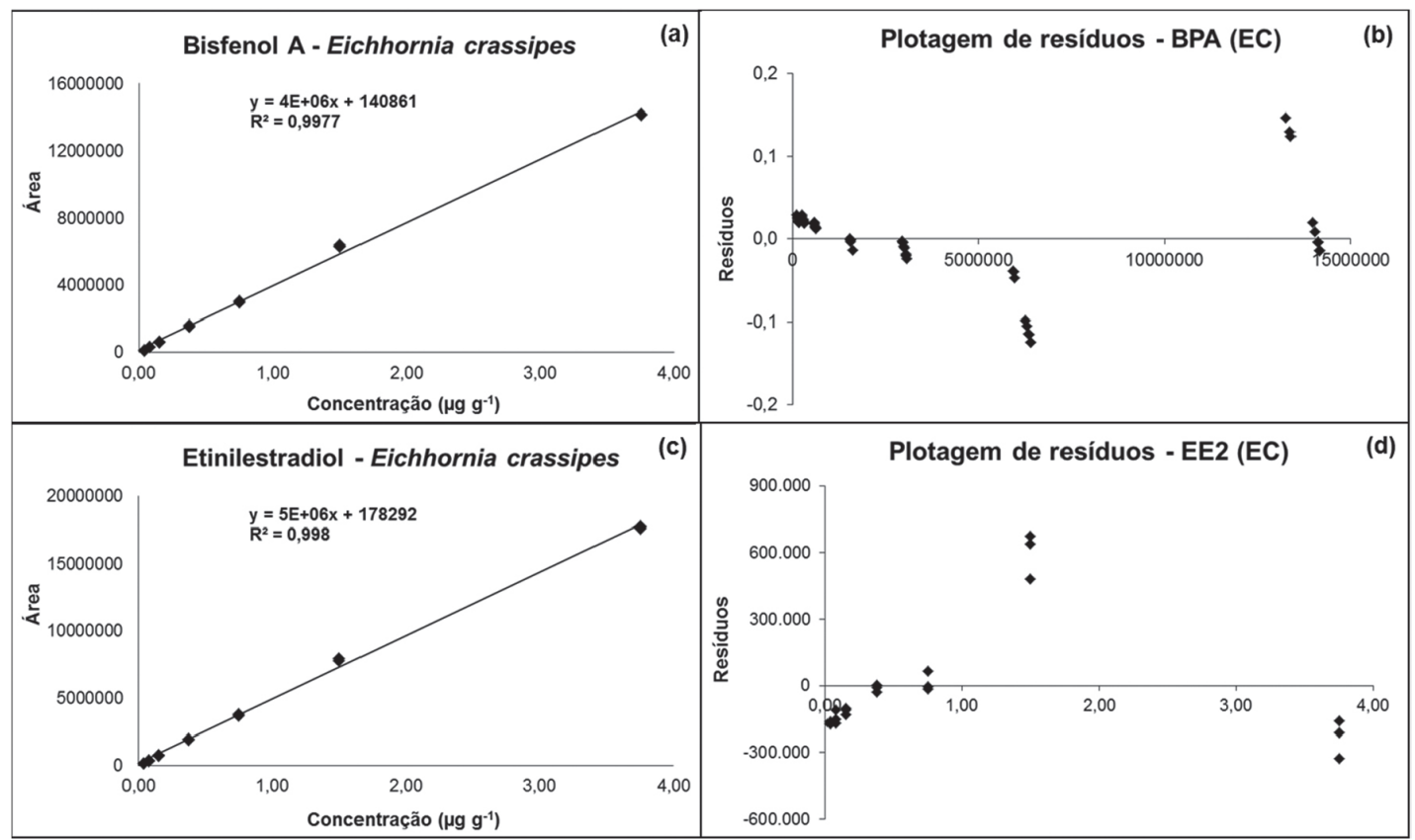

Figura 5. Curva analítica de BPA (a) e plotagem dos resíduos de BPA (b), e curva analítica de EE2 (c) e plotagem dos resíduos de EE2 (d) em aguapé

Tabela 2. Estatística da regressão linear

\begin{tabular}{lcccc}
\hline \multicolumn{4}{c}{ Estatística de regressão } \\
\hline R múltiplo & BPA (aguapé) & EE2 (aguapé) & BPA (mini-papiro) & EE2 (mini-papiro) \\
R-quadrado & 0,998867 & 0,998979 & 0,995265 & 0,999084 \\
R-quadrado ajustado & 0,997735 & 0,997958 & 0,990552 & 0,998169 \\
Erro padrão & 0,997610 & 0,997845 & 0,989825 & 0,998054 \\
Observações & 238353,5 & 282804,6 & 848958,6 & 256154,6 \\
\hline
\end{tabular}

para EE2. ${ }^{39}$ Os LD atingidos pelos autores foram $0,39 \mathrm{ng} \mathrm{g}^{-1}$ para BPA e 2,68 $\mathrm{ng} \mathrm{g}^{-1}$ para EE2, e os LQ foram $1,30 \mathrm{ng} \mathrm{g}^{-1}$ para BPA e $12,88 \mathrm{ng} \mathrm{g}^{-1}$ para EE2. Os autores utilizaram $0,5 \mathrm{~g}^{\text {de }}$ amostra e
$25 \mathrm{~mL}$ de 75:25 $\mathrm{H}_{2} \mathrm{O}: \mathrm{MeOH}$ na extração em micro-ondas, e o extrato foi posteriormente diluído a $5 \%$ de $\mathrm{MeOH}$ para a realização da SPE. A eluição foi realizada com $4 \mathrm{~mL}$ de $\mathrm{MeOH}$, os extratos secos em 
Tabela 3. Análise de variância da linearidade para mini-papiro

\begin{tabular}{|c|c|c|c|c|c|}
\hline \multicolumn{6}{|c|}{ Bisfenol A } \\
\hline & Graus de liberdade & Soma quadrática & Média quadrática & Estatística $F$ & $F$ de significância \\
\hline Regressão & 1 & $9,82325 \mathrm{E}+14$ & $9,82325 \mathrm{E}+14$ & 1362,96 & $1,51 \mathrm{E}-14$ \\
\hline Resíduo & 13 & $9,3695 \mathrm{E}+12$ & $7,20731 \mathrm{E}+11$ & & \\
\hline Total & 14 & $9,91694 \mathrm{E}+14$ & & & \\
\hline \multicolumn{6}{|c|}{ Etinilestradiol } \\
\hline & Graus de liberdade & Soma quadrática & Média quadrática & Estatística $F$ & $F$ de significância \\
\hline Regressão & 1 & $5,72291 \mathrm{E}+14$ & $5,72291 \mathrm{E}+14$ & 8721,93 & $2,48 \mathrm{E}-23$ \\
\hline Resíduo & 16 & $1,04984 \mathrm{E}+12$ & 65615158719 & & \\
\hline Total & 17 & $5,73341 \mathrm{E}+14$ & & & \\
\hline
\end{tabular}

Tabela 4. Análise de variância da linearidade para aguapé

\begin{tabular}{|c|c|c|c|c|c|}
\hline \multicolumn{6}{|c|}{ Bisfenol A } \\
\hline & Graus de liberdade & Soma quadrática & Média quadrática & Estatística $F$ & $F$ de significância \\
\hline Regressão & 1 & $4,50543 \mathrm{E}+14$ & $4,50543 \mathrm{E}+14$ & 7930,36 & $2,90851 \mathrm{E}-25$ \\
\hline Resíduo & 18 & $1,02262 \mathrm{E}+12$ & 56812398208 & & \\
\hline Total & 19 & $4,51566 \mathrm{E}+14$ & & & \\
\hline \multicolumn{6}{|c|}{ Etinilestradiol } \\
\hline & Graus de liberdade & Soma quadrática & Média quadrática & Estatística $F$ & $F$ de significância \\
\hline Regressão & 1 & $7,03702 \mathrm{E}+14$ & $7,03702 \mathrm{E}+14$ & 8798,64 & $1,14 \mathrm{E}-25$ \\
\hline Resíduo & 18 & $1,43961 \mathrm{E}+12$ & 79978444868 & & \\
\hline Total & 19 & $7,05141 \mathrm{E}+14$ & & & \\
\hline
\end{tabular}

Tabela 5. Limites de detecção e quantificação do método para mini-papiro e aguapé

\begin{tabular}{lccccc}
\hline \multirow{2}{*}{ Interferente endócrino } & \multicolumn{3}{c}{ Mini-papiro } & & \multicolumn{2}{c}{ Aguapé } \\
\cline { 2 - 3 } \cline { 5 - 6 } & $\mathrm{LD}^{(1)}\left(\mu \mathrm{g} \mathrm{g}^{-1}\right)$ & $\mathrm{LQ}^{(2)}\left(\mu \mathrm{g} \mathrm{g}^{-1}\right)$ & $\mathrm{LD}^{(1)}\left(\mu \mathrm{g} \mathrm{g}^{-1}\right)$ & 0,15 \\
Bisfenol A & 0,15 & 1,50 & 0,045 & $\mathrm{LQ}^{(2)}\left(\mu \mathrm{g} \mathrm{g}^{-1}\right)$ \\
Etinilestradiol & 0,045 & 0,15 & 0,15 & 0,75 \\
\hline
\end{tabular}

(1)limite de detecção do método; (2)limite de quantificação do método.

nitrogênio e ressuspendidos em $500 \mu \mathrm{L}$ de 80:20 $\mathrm{H}_{2} \mathrm{O}: \mathrm{MeOH}$. Os LDI, LQI, LD e LQ obtidos pelos autores, ${ }^{39}$ inferiores ao presente estudo podem ser devido ao menor volume final de amostra obtida $(500 \mu \mathrm{L})$ em comparação aos $3 \mathrm{~mL}$ obtidos nesse estudo. Porém, a vantagem de usar o FL é que é um equipamento de menor custo e de uso mais simples, sendo disponível em maior número nos laboratórios.

A análise de EE2 em plantas de feijão extraídas em acetonitrila e água, com posterior clean-up em cartucho C18 de extração em fase sólida, e leitura realizada em HPLC-UV e em coluna C18 foi realizada por Karnjanapiboonwong et al., ${ }^{40}$ que obtiveram limites de detecção de $0,1 \mu \mathrm{g} \mathrm{g}^{-1}$ de EE2. Observa-se que os limites encontrados pelos autores são bem próximos aos encontrados neste estudo.

\section{Exatidão (recuperação)}

A exatidão do método foi avaliada por meio de ensaios de recuperação adicionando-se concentrações conhecidas dos analitos em macrófitas isentas dos mesmos. Ensaios de recuperação devem ser realizados em um nível de concentração próximo ao limite de quantificação do método, e pelo menos em mais um nível acima desse, com no mínimo 5 ensaios no total. ${ }^{38}$ Para BPA no aguapé foram adicionados três diferentes níveis de concentração, sendo $0,15 \mu \mathrm{g} \mathrm{g}{ }^{-1}$,
$0,75 \mu \mathrm{g} \mathrm{g}^{-1}$ e $1,5 \mu \mathrm{g} \mathrm{g}^{-1}$. Para EE2 no aguapé as recuperações foram feitas nos níveis de concentrações $0,75 \mu \mathrm{g} \mathrm{g}^{-1}$ e $1,5 \mu \mathrm{g} \mathrm{g}^{-1}$, num total de 6 ensaios injetados em triplicata.

Para BPA no mini-papiro as concentrações utilizadas foram 1,5 $\mu \mathrm{g} \mathrm{g}^{-1}$ e 3,0 $\mu \mathrm{g} \mathrm{g}^{-1}$, em um total de 9 ensaios injetados em triplicata. Para EE2 no mini-papiro os níveis de concentração utilizados foram $0,15 \mu \mathrm{g} \mathrm{g}^{-1}, 0,75 \mu \mathrm{g} \mathrm{g}^{-1}, 1,5 \mu \mathrm{g} \mathrm{g} \mathrm{g}^{-1}$ e $3,0 \mu \mathrm{g} \mathrm{g} \mathrm{g}^{-1}$. Na Tabela 6 estão apresentados os resultados de recuperação de BPA e EE2 no aguapé e no mini-papiro. Os valores de recuperação permaneceram entre 70 e $120 \%$ e, portanto, a exatidão do método ficou dentro dos níveis aceitáveis. ${ }^{38}$

Analisando BPA em amostras de alface foi obtida recuperação de $61,1 \%$ de BPA na concentração de $0,3 \mu \mathrm{g} \mathrm{g}^{-1}$, ${ }^{41}$ inferior às obtidas no presente estudo, e recuperação teórica de $76 \% .{ }^{41}$ Uma recuperação média de 71,9 $\pm 2,3 \%$ de EE2 foi obtida na concentração de $1 \mu \mathrm{g} \mathrm{g}^{-1}$ em plantas de feijão. ${ }^{40}$

Em amostras de P. australis proveniente de wetlands construídas que tratavam esgoto municipal secundário no Reino Unido os autores obtiveram recuperações de $105,0 \%, 124,9 \%$ e $149,7 \%$ nos níveis baixo, médio e alto, respectivamente, para BPA. ${ }^{39}$ Para EE2 foram relatadas recuperações de $81,2 \%, 49,5 \%$ e $70,9 \%$ para os níveis baixo, médio e alto, respectivamente, e a maioria dos compostos estudados obtiveram recuperações na faixa de $80-120 \% .{ }^{39}$ Os autores estudaram 
Tabela 6. Recuperações médias obtidas na validação para aguapé e mini-papiro

\begin{tabular}{|c|c|c|c|c|c|c|c|}
\hline \multirow{3}{*}{$\begin{array}{l}\text { Interferente } \\
\text { endócrino }\end{array}$} & \multicolumn{7}{|c|}{ Recuperação (\%) } \\
\hline & \multicolumn{3}{|l|}{ Aguapé } & \multicolumn{4}{|c|}{ Mini-papiro } \\
\hline & $\begin{array}{c}0,15 \\
\left(\mu \mathrm{g} \mathrm{g}^{-1}\right)\end{array}$ & $\begin{array}{c}0,75 \\
\left(\mu g g^{-1}\right)\end{array}$ & $\begin{array}{c}1,50 \\
\left(\mu \mathrm{g} \mathrm{g}^{-1}\right)\end{array}$ & $\begin{array}{c}0,15 \\
\left(\mu g g^{-1}\right)\end{array}$ & $\begin{array}{c}0,75 \\
\left(\mu \mathrm{g} \mathrm{g}^{-1}\right)\end{array}$ & $\begin{array}{c}1,50 \\
\left(\mu \mathrm{g} \mathrm{g}^{-1}\right)\end{array}$ & $\begin{array}{c}3,00 \\
\left(\mu g^{-1}\right)\end{array}$ \\
\hline Bisfenol A & 102,7 & 98,1 & 96,9 & ---- & ---- & 119,4 & 96,7 \\
\hline Etinilestradiol & ---- & 115,9 & 113,7 & 83,5 & 93,2 & 95,7 & 102,8 \\
\hline
\end{tabular}

Tabela 7. Repetitividade média do método analítico para aguapé e mini-papiro

\begin{tabular}{|c|c|c|c|c|c|c|c|}
\hline \multirow{3}{*}{$\begin{array}{l}\text { Interferente } \\
\text { endócrino }\end{array}$} & \multicolumn{7}{|c|}{ Repetitividade - coeficiente de variação (\%) } \\
\hline & \multicolumn{3}{|l|}{ Aguapé } & \multicolumn{4}{|c|}{ Mini-papiro } \\
\hline & $\begin{array}{c}0,15 \\
\left(\mu g^{-1}\right)\end{array}$ & $\begin{array}{c}0,75 \\
\left(\mu g^{-1}\right)\end{array}$ & $\begin{array}{c}1,50 \\
\left(\mu g^{-1}\right)\end{array}$ & $\begin{array}{c}0,15 \\
\left(\mu g g^{-1}\right)\end{array}$ & $\begin{array}{c}0,75 \\
\left(\mu g^{-1}\right)\end{array}$ & $\begin{array}{c}1,50 \\
\left(\mu g^{-1}\right)\end{array}$ & $\begin{array}{c}3,00 \\
\left(\mu g g^{-1}\right)\end{array}$ \\
\hline Bisfenol A & 4,1 & 1,8 & 0,3 & ---- & ---- & 1,9 & 1,0 \\
\hline Etinilestradiol & ---- & 0,2 & 0,4 & 6,2 & 2,0 & 0,9 & 1,0 \\
\hline
\end{tabular}

Tabela 8. Precisão intermediária média do método analítico para aguapé e mini-papiro

\begin{tabular}{|c|c|c|c|c|c|c|c|}
\hline \multirow{3}{*}{$\begin{array}{l}\text { Interferente } \\
\text { endócrino }\end{array}$} & \multicolumn{7}{|c|}{ Precisão intermediária - coeficiente de variação (\%) } \\
\hline & \multicolumn{3}{|c|}{ Aguapé } & \multicolumn{4}{|c|}{ Mini-papiro } \\
\hline & $\begin{array}{c}0,15 \\
\left(\mu g^{-1}\right)\end{array}$ & $\begin{array}{c}0,75 \\
\left(\mu \mathrm{g} \mathrm{g}^{-1}\right) \\
\end{array}$ & $\begin{array}{c}1,50 \\
\left(\mu \mathrm{g} \mathrm{g}^{-1}\right)\end{array}$ & $\begin{array}{c}0,15 \\
\left(\mu g^{-1}\right) \\
\end{array}$ & $\begin{array}{c}0,75 \\
\left(\mu \mathrm{g} \mathrm{g}^{-1}\right)\end{array}$ & $\begin{array}{c}1,50 \\
\left(\mu \mathrm{g} \mathrm{g}^{-1}\right) \\
\end{array}$ & $\begin{array}{c}3,00 \\
\left(\mu g g^{-1}\right) \\
\end{array}$ \\
\hline Bisfenol A & 3,9 & 1,4 & 0,5 & ---- & ---- & 1,7 & 1,0 \\
\hline Etinilestradiol & ---- & 0,3 & 0,3 & 5,8 & 1,5 & 1,2 & 1,0 \\
\hline
\end{tabular}

no total 81 compostos, mas algumas das recuperações obtidas pelos autores ficaram fora da faixa de $70-120 \% .^{38}$

\section{Precisão (repetitividade e precisão intermediária)}

A precisão foi obtida por meio da determinação do coeficiente de variação das replicatas no mesmo dia (repetitividade) e em dias diferentes (precisão intermediária). Como podem ser observados na Tabela 7, os coeficientes de variação (CV) das amostras ficaram abaixo de $20 \%$, indicando que o método possui repetitividade adequada. ${ }^{38}$ Os valores de precisão intermediária foram obtidos ao longo do tempo, e os coeficientes de variação sempre ficaram dentro das faixas aceitáveis, como podem ser observados na Tabela 8 para BPA e EE2, nas macrófitas aguapé e mini-papiro.

\section{Análises de amostras reais de mini-papiro e de aguapé}

Amostras provenientes de wetlands construídas de mini-papiro e de aguapé foram analisadas e os valores encontrados foram nas faixas <LQ a 1,1 $\mu \mathrm{g} \mathrm{g}^{-1}$ no aguapé (tanto para BPA quanto para EE2), de $<$ LQ a 2,2 $\mu \mathrm{g} \mathrm{g}^{-1}$ de BPA no mini-papiro, e de $<\mathrm{LQ}$ a $0,8 \mu \mathrm{g} \mathrm{g}^{-1}$ de EE2 no mini-papiro, demonstrando que o método desenvolvido e validado foi adequado para o uso pretendido. Com estes resultados foi possível confirmar a absorção dos compostos pelas plantas utilizadas, ajudando a comprovar que um dos mecanismos de remoção destes interferentes endócrinos em wetlands construídas é absorção pelas macrófitas. Como existem outros possíveis mecanismos de retenção, como adsorção pelo carvão e biodegradação pelos microrganismos presentes no sistema, não é possível avaliar a taxa de remoção dos IE pelas plantas. Entretanto, o conhecimento deste mecanismo é importante para estabelecer o descarte/destino das plantas utilizadas nas wetlands construídas, bem como a necessidade de renovação das plantas no sistema.

\section{CONCLUSÕES}

O método analítico desenvolvido e validado para quantificação de BPA e EE2 em amostras de mini-papiro e aguapé mostrou ser eficiente, sensível e adequado, atendendo a todos os requisitos estabelecidos pelos guias de validação: seletividade, linearidade e faixa linear de trabalho, sensibilidade, exatidão (recuperação) e precisão (repetitividade e precisão intermediária). ${ }^{37,38} \mathrm{O}$ método foi utilizado com sucesso nos estudos de absorção desses compostos nas duas espécies de plantas utilizadas neste trabalho, provenientes de wetlands construídas e também pode ser útil para estudos de fitorremediação desses compostos (BPA e EE2) por essas espécies.

\section{AGRADECIMENTOS}

Os autores agradecem à CAPES e ao CNPq (Processo n. ${ }^{\circ}$ 149364/2014-8) pelas bolsas de doutorado e taxa de bancada, ao Fundo de Apoio ao Ensino, Pesquisa e Extensão (FAEPEX) da UNICAMP, pelos auxílios financeiros concedidos, à Embrapa Meio Ambiente e à Faculdade de Engenharia Agrícola da UNICAMP.

\section{REFERÊNCIAS}

1. Wee, S. Y.; Aris, A. Z.; Environ. Int. 2017, 6, 207.

2. United States Environmental Protection Agency; Special report on environmental endocrine disruption: an effects assessment and analysis. EPA/630/R-96/012, 1997.

3. Kim, S. D.; Cho, J.; Kim, I. S.; Vanderford, B. J.; Snyder, S. A.; Water Res. 2007, 41, 1013.

4. Migeot, V.; Dupuis, A.; Cariot, A., Albouy-Llaty, M.; Pierre, F.; Rabouan, S. Environ. Sci. Technol.; 2013, 47, 13791.

5. Brasil. Agência Nacional de Vigilância Sanitária. Resolução RDC n. ${ }^{\circ} 41$, de 16 de setembro de 2011. Dispõe sobre a proibição de uso de bisfenol 
A em mamadeiras destinadas a alimentação de lactentes e dá outras providencias. Diário Oficial da União. Poder Executivo. 2011.

6. Tran, N. H.; Gin, K. Y. H.; Sci. Total Environ. 2017, 599 - 600, 1503.

7. Froehner, S.; Piccioni, W.; Machado, K. S.; Aisse, M. M.; Water Air Soil Pollut. 2011, 216, 463.

8. Diniz, M. S.; Maurício, R.; Petrovic, M.; De Alda, M. J. L.; Amaral, L.; Peres, I.; Barceló, D.; Santana, F.; J. Environ. Sci. 2010, 22, 1613.

9. Montagner, C. C.; Jardim, W. F.; J. Braz. Chem. Soc. 2011, 22, 1452.

10. Herrera-Melían, J. A.; Guedes-Alonso, R.; Borreguero-Fabelo, A.; Santana-Rodríguez, J. J.; Sosa-Ferrera, Z.; Environ. Sci. Pollut. Res. 2017.

11. Gray, J. L.; Sedlak, D. L.; Water Environ. Res. 2005, 77, 24.

12. Kumar, A. K.; Chiranjeevi, P.; Mohanakrishna, G.; Mohan, S. V.; Ecol. Eng. 2011, 37, 1555.

13. King, O. C.; Van De Merwe, J. P.; McDonald, J. A.; Leusch, F. D. L.; Environ. Toxicol. Chem. 2016, 35, 1378.

14. Grover, D. P.; Zhou, J. L.; Frickers, P. E.; Readman, J. W.; J. Hazard. Mater. 2011, 185, 1005.

15. Hintemann, T.; Schneider, C.; Schöler, H. F.; Schneider, R.; J. Water Res. 2006, 40, 2287.

16. United States Environmental Protection Agency; The third unregulated contaminant monitoring rule (UCMR3). EPA 815-F-12-004, 2012.

17. Pojana, G.; Gomiero, A.; Jonkers, N.; Marcomini, A.; Environ. Int. 2007, 33, 929.

18. Yan, Q.; Min, J.; Yu, Y.; Zhu, Z.; Feng, G.; Chemosphere 2017, 186, 823.

19. Xiong, J. Q.; Kurade, M. B.; Jeon, B. H.; Trends Biotechnol. 2018, 36, 30.

20. Sophia, A. C.; Lima, E. C.; Ecotoxicol. Environ. Saf. 2018, 150, 1.

21. Vymazal, J.; Sci. Total Environ. 2007, 380, 48.

22. Deutsche Gesellschaft für Internationale Zusammenarbeit $\mathrm{GmbH}$; Technology review of constructed wetlands: Subsurface flow constructed wetlands for greywater and domestic wastewater treatment. Sustainable sanitation - ecosan program. 2011.

23. Hijosa-Valsero, M.; Matamoros, V.; Sidrach-Cardona, R.; MartínVillacorta, J.; Bécares, E.; Bayona, J. M.; Water Res. 2010, 44, 3669.

24. He, Y.; Nurul, S.; Schmitt, H.; Sutton, N. B.; Murk, T. A. J.; Blokland, M. H.; Rijnaarts, H. H. M.; Langenhoff, A. A. M.; Sci. Total Environ. 2018, 631, 1572

25. Dai, Y.-N.; Tao, R.; Tai, Y.-P.; Tam, N. F.-Y.; Dan, A.; Yang, Y.; Ecological Engineering 2017, 99, 496.
26. Toro-Vélez, A. F.; Madera-Parra, C. A.; Peña-Varón, M. R.; Lee, W. Y.; Bezares- Cruz, J. C.; Walker, W. S.; Cárdenas-Henao, H.; QuesadaCalderón, S.; García- Hernández, H.; Lens, P. N. L. Sci. Total Environ. 2016, 542,93 .

27. Papaevangelou, V. A.; Gikas, G. D.; Tsihrintzis, V. A.; Antonopoulou, M.; Konstantinou, I. K. Chemical Engineering Journal. 2016, 294, 146.

28. Ávila, C.; Bayona, J. M.; Martín, I.; Salas, J. J.; García, J. Ecological Engineering. 2015, 80, 108.

29. Vymazal, J.; Březinová, T. D.; Koželuh, M.; Kule, L. Ecological Engineering. 2017, 98, 354.

30. Carranza-Diaz, O.; Schultze-Nobre, L.; Moeder, M.; Nivala, J.; Kuschk, P.; Koeser, H. Ecological Engineering. 2014, 71, 234.

31. Li, G.; Zhai, J.; He, Q.; Zhi, Y.; Xiao, H.; Rong, J. Journal of Environmental Sciences. 2014, 26, 1869.

32. Ávila, C.; Matamoros, V.; Reyes-Contreras, C.; Piña, B.; Casado, M.; Mita, L.; Rivetti, C.; Barata, C.; García, J.; Bayona, J. M. Sci. Total Environ. 2014, 470, 1272.

33. Chen, T-C.; Yeh, K-J. C.; Kuo, W-C.; Chao, H-R.; Sheu, S-C. J. Hazard. Mater. 2014, 277, 62.

34. Campos, J. M.; Tese de Doutorado, Universidade Estadual de Campinas, Brasil, 2018.

35. Boaretto, A. E.; Van Raij, B.; Silva, F. C.; Chitolina, J. C.; Tedesco, M. J.; Carmo, C. A. F. S. Manual de análises químicas de solos, plantas e fertilizantes. 2nd. ed., Brasília: Embrapa Informação Tecnológica, 2009.

36. Campos, J. M.; Queiroz, S. C. N.; Roston, D. M. Resumos do $29^{\circ}$ Congresso Brasileiro de Engenharia Sanitária e Ambiental. São Paulo, Brasil, 2017.

37. Inmetro. Instituto Nacional de Metrologia, Normalização e Qualidade Industrial. Orientação sobre validação de métodos analíticos. DOQCGCRE-008. Revisão 07 - Julho de 2018.

38. SANTE/11813/2017. Guidance document on analytical quality control and method validation procedures for pesticide residues and analysis in food and feed. Supercedes SANTE/11945/2015. Implemented by 01/01/2018.

39. Petrie, B.; Smith, B. D. B. D.; Youdan, J.; Barden, R.; KasprzykHordern, B. Anal. Chim. Acta. 2017, 959, 91.

40. Karnjanapiboonwong, A.; Chase, D. A.; Cañas, J. E.; Jackson, W. A.; Maul, J. D.; Morse, A. N.; Anderson, T. A. Ecotoxicol. Environ. Saf. 2011, 74, 1336.

41. Calderón-Preciado, D.; Jiménez-Cartagena, C.; Peñuela, G.; Bayona, J. M. Anal. Bioanal. Chem. 2009, 394, 1319. 